\title{
On the Impact of Sequence and Time in Rich Media Advertising
}

\author{
Benoit Baccot, Omar Choudary, Romulus Grigoras, Vincent Charvillat \\ University of Toulouse, IRIT-ENSEEIHT, France \\ \{bbaccot, omar.choudary, grig, charvi\}@enseeiht.fr
}

\begin{abstract}
Nowadays, commercial websites use an increasing amount of rich media ads, since these ads grab users' attention more easily. Ad overloading has a counterproductive effect and we advocate a more parsimonious and finely-tuned use of ads. In this paper we stress the importance of using ads in the right sequence and at the right time. We also present how to use our web adaptation system for a classic web marketing multivariable test. The experimental results show the usefulness of optimizing banners' sequences and timing.
\end{abstract}

\section{Categories and Subject Descriptors}

D.2.11 [Software Engineering]: Software Architectures

\section{General Terms}

Algorithms, Human factors, Performance

\section{Keywords}

Rich media advertiving, Banner sequences, Advertisement impact

\section{INTRODUCTION}

Web site adaptation to the user's context is paramount in providing the user with an optimal experience. For example, recommending additional interesting information in the form of a banner can help the user find more quickly what he is looking for on a given site. On a commercial website, contextual advertisement on a banner is used to drive users and transform their navigation into a transaction. Traditionally, the recommended information on a banner has been presented in the form of text and hyperlinks. Recent studies [7] have shown the benefits of using rich media for displaying motion and exploit sensory information such as video, audio, animation etc. Rich media content is considered more attractive since it can grab users' attention easily and can also leave stronger memories. These are some of the

Permission to make digital or hard copies of all or part of this work for personal or classroom use is granted without fee provided that copies are not made or distributed for profit or commercial advantage and that copies bear this notice and the full citation on the first page. To copy otherwise, to republish, to post on servers or to redistribute to lists, requires prior specific permission and/or a fee.

MM'09, October 19-24, 2009, Beijing, China.

Copyright 2009 ACM 978-1-60558-608-3/09/10 ...\$10.00. reasons that made rich media advertising very popular. Unfortunately, some sites make excessive use of it, and this is commonly called "the ad overload problem".

Recent research has studied the selection of content to be displayed as recommendation or as an advertisement. Nevertheless, little effort has been put into optimizing the use of rich media. Challenging questions need answers: for a given informational content, what is the most appropriate presentation (textual, visual, audio)? How much interactivity? What is the benefit of using immersive or 3D content? How can we evaluate a sequence of banners? What are the optimal moments for presenting a banner?

The rest of this paper is organized as follows. First we state the problem and introduce our argument. The following section presents some pointers from the literature of a large research field: rich media web advertising. Section 4 gives an overview of the experimental platform used for the multivariate test presented in section 5. Finally, we conclude and provide perspectives of this work.

\section{PROBLEM STATEMENT}

We think that sequential advertising [4] can be a solution for the ad overload problem. Indeed, some web sites today make excessive use of rich media, perceptually aggressive advertisements. Instead of having a set of banners competing for user attention at the same time, it is more efficient to follow a users' navigation sequence and provide users with a sequence of banners, in the right form, at the right time.

This paper presents a system that can help a web site owner to understand the impact of his advertisement policies and be able to fine tune them. Our software platform is able to finely capture the navigation context of a user, build a high-level navigation metric: an activity level, implement an advertisement policy on a fine timeline and also observe its impact on the activity level of the user. The activity level is an example of a success criteria the webmaster can target. Our experimental results show that the banner sequence, the format and the timing influence the advertisement impact, which allows us to infer on the best advertisement strategies.

In this paper we also argue that the impact of rich media banners should be studied in its multi-dimensionality: the classical dimensions of informational content but also the format, the sequentiality and the timeline, w.r.t. the timeline of the web user's navigation. These dimensions sequence, timing (interactivity, animation) and banner format - all share one common trait in that they refer to nonmessage aspects of the banner. Our results confirm two observations: an improper format of a banner in the beginning 
of a banner sequence wipes out the positive effect of the subsequent banners ; the improper timing of displaying the banner results in a reduced banner impact.

\section{STATE OF THE ART}

A potential barrier to banners effectiveness is the volume of banners to which consumers are exposed [7]. Consumers often limit their information exposures by engaging in selective perception, by which they process a limited number of banners and ignore others. Some systems have been built in order to include ads in existing web page components such as images [5] or video sequences.

Researchers often use A/B tests to carry out experiments on the web [1]. They segment users in two groups (A and B) to which two web content versions are presented and finally observe which one is the best. Our software platform can help to conduct A/B tests such as [8], in which interactivity is compared with other structural features of online ads such as animation and ad shape.

Some researchers confirm that ad sequence matters [3]. They differentiate between publicity (unpaid advertising, often in the form of personal recommendations or spreading buzz) and advertising. Authors test whether the sequencing, or order of presentation, of publicity and advertising influences message processing. Results suggest that the sequence, publicity-then-advertising, is most effective at persuading potential customers to visit a tourist destination.

The sequence is also important when it comes to the overall emotional impact of an ad sequence. In [2], authors show that when emotions are experienced, positive emotions facilitate coping with later negativity, and an ad with declining (vs. improving) emotions results in more favorable attitudes.

In order to implement effective advertising campaigns, web analytics tools have been proposed by both the research community and the industry. Some tools (e.g. PhpMyVisit) capture web usage information. Others are specifically designed for mining this wealth of information (e.g. Clementine). Finally, ad servers such as OpenX propose frameworks to manage online advertisement inclusion into web sites.

Conventional advertisement techniques lack a timecontrol function for advertisement insertion, so the advertisement exposure is not optimal. Takashi et al [6] propose a technique in which advertisement insertion control is based on the history of each viewer. Their system controls the insertion of streaming advertisements into streaming content. The system is similar to ours in that it decides of the moment an ad is inserted, it chooses the ad and collects feedback information. However, the system is specific to streaming media and only works inside media players.

\section{OUR EXPERIMENTAL PLATFORM}

We have developed a generic adaptation platform that we use for our study. Our system is able to dynamically adapt website pages during a browsing session. Adaptation can be done in different ways: add or update of the web page content, change in the presentation or the structure etc. The platform has three main components (figure 1). First the user behavior is observed and his interactions are collected. Interactions (called events) are sent back to the server and analyzed in order to decide whether an adaptation should be done or not. Another component, called Effector, performs the web page adaptation as decided by the Analyzer.

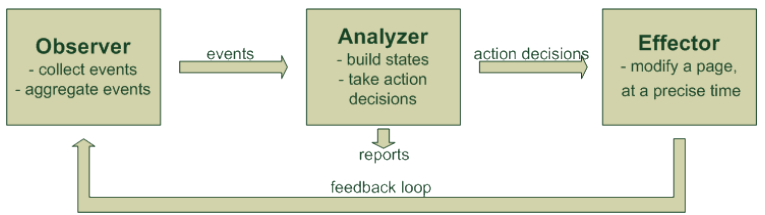

Figure 1: Platform overview

The system works in a closed loop: when an adaptation is performed by the Effector, the user continues to be observed and the effect of the adaptation is measured by the Analyzer.

The Observer captures user browsing events and is composed of two parts: the event logger (server-side) receives events from the event collector (client side). The client side component captures Javascript/HTML DOM events: all mouse, key and scroll events. VCR events from embedded multimedia objects (video players, Flash applications) are also collected. As some events may be very frequent, we have included an aggregator: instead of sending all events individually to the server, we compact them into a special event that sums up the information (e.g. if lots of scroll events are detected on a page, they can be compacted into a higher level event describing the visited part of the page).

The Analyzer interprets the events and builds a state for each connected user. This state describes the context (already visited pages, user activity level on the site) and is updated as often as needed. When a new state is computed, the analyzer can either dynamically decide of an adaptation action or apply a predefined adaptation strategy. The strategy can be static (e.g. defined by a marketer), or dynamically learnt by the system (e.g. using reinforcement learning). Like other web analytics solutions, our Analyzer can classify users in different segments, and apply differentiated strategies. Based on this segmentation, it is able to generate reports at different levels of granularity (global population, targeted groups, individuals or sessions for a given user), allowing us to conduct A/B tests.

Finally, the Effector applies the adaptation decisions chosen by the analyzer. Like the Observer, it contains two components: one running in the client web browser (implemented in Javascript), periodically asking the one running on the server if an action decision is available for the current user. If so, a node providing the HTML ad insertion code is added to the DOM tree of the page. The specificity of the Effector is its ability to dynamically modify a page, while a user surfing it, without the need to reload it. As a result, we can finely determine when an action should be performed during the user navigation.

\section{AN A/B TEST FOR MEASURING BAN- NERS IMPACT}

In order to support our argument that sequence and timing are important for the effectiveness of banners, we conduct an A/B test: we test variations of banner sequences and timing by exposing a test site to a randomly selected set of users in a live experiment.

\subsection{Test website}

We designed a standard, informative website describing activities (entertainment, shops and restaurants) available 

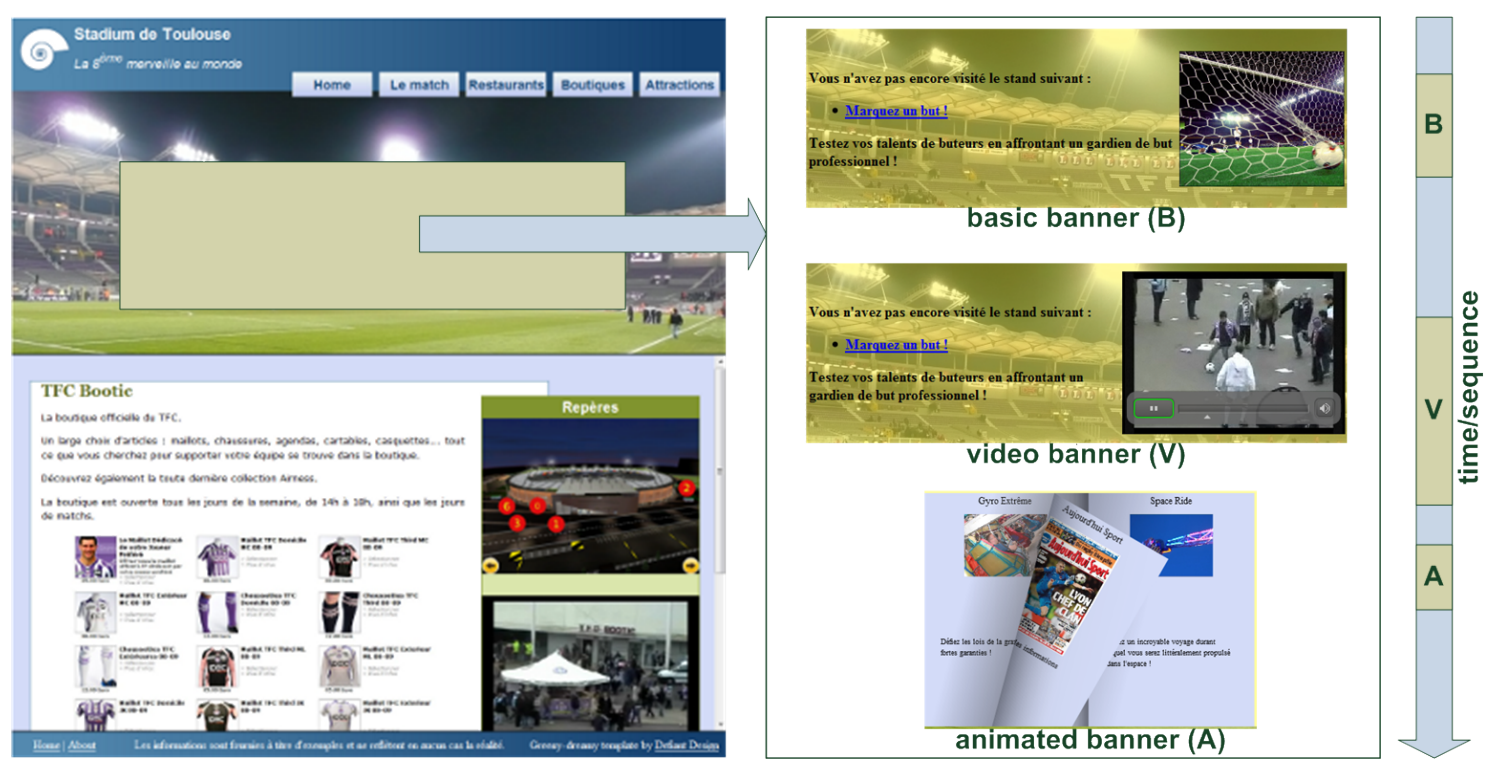

Figure 2: The test website and the three types of banners

before and after a match around the stadium of Toulouse (figure 2). Each activity is presented on a single page, using various types of media, from the simplest to the richest: a text description, an image, a Flash video, a map and a $3 \mathrm{D}$ view of the stadium, both including clickable points of interest corresponding to the closest activities.

Additionally, we designed three types of banners (figure 2) for each activity. The basic banner (B) is composed of some text and an image, both describing a given activity. The video banner $(\mathrm{V})$ is composed of some text and a video. The animated banner (A) is represented as a book, where each page describes an activity (using some text and an image). The user can interact with this banner and can turn the pages as if it was a real book. All banners are clickable, and redirect to the proposed content page.

We deploy our adaptation platform on the test site and conduct the A/B test. Users are randomly divided into seven segments using their HTTP session identifier. For each segment, just like a web marketer would proceed, we assign a sequence of three banners to display, except for a special segment that would not receive any banners. The content of the banners presents an activity that the current user has not yet visited on the website. Using the Analyzer reports, we are thus able to compare key metrics for each segment.

Banners are displayed following the sequence associated to the user segment. In order to identify the time when a banner should be injected on a page, the Analyzer computes the activity level of the current user. Activity is defined as the number of events produced over a given time window. When the Analyzer detects a significant decrease of user activity, it decides to inject a banner, according to the user segment and its associated sequence. When all three banners have been displayed, the Analyzer is turned off.

Figure 3 presents the activity for two users from segments $B A V$ and $V B A$ and points out the moments when the banner is injected (following a decrease of the activity level) and its impact (feedback information).

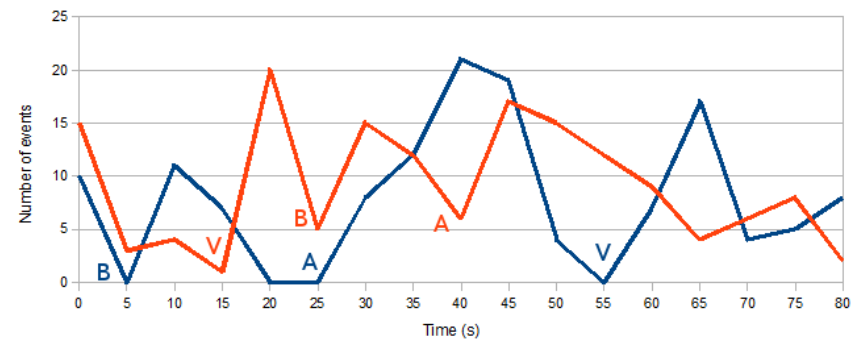

Figure 3: The impact of banners on user activity for two individual browsing sessions

\subsection{Test Protocol}

We asked individuals to visit the test site. On the welcome page, a text gives the context of the test site (animations around the stadium), encourages users to discover the different pages, and choose the one they prefer. When they have chosen, they can fill a form. After validating, they get a thank message and are encouraged to give the site URL to their friends/family members.

We conducted three test rounds that allowed us to study the impact of the time when banners are inserted (A/B test for the timing). We also used the first round for studying into more detail the impact of the banner sequence (A/B test for the sequence).

\subsection{Results}

In order to get as many test users as possible, we sent the website URL to our entourage by email. We also asked students from our university and publicized the test on social networks. We managed to get 169 testers for which we logged the 94808 events they produced on the test website.

For the first test round, the Analyzer was configured to insert the first banner upon the first activity decrease. While interpreting the results, we realized that the key met- 
rics that we had initially targeted (e.g. average session length and standard deviation) could not provide us with results. It proved to be that these metrics are very sensible to the noise inherent in web sessions. Therefore we chose to use a metric that can help filter out invalid data and computed a table using quintiles (5-quantiles) (table 4). Each column represents the rank of each group according to the session duration for a given quintile (rank 1 corresponds to the shortest session, rank 7 to the longest). A quintile represents $20 \%$ of a given amount: in our case, we sort all sessions for a given group w.r.t. the duration, and we take the one corresponding to $20 \%$ of the number of sessions for this group.

\begin{tabular}{|c|c|c|c|c|}
\hline Rank & $20 \%$ & $40 \%$ & $60 \%$ & $80 \%$ \\
\hline 1 & ABV & ABV & ABV & ABV \\
2 & BAV & AVB & AVB & AVB \\
3 & BVA & VBA & N & N \\
4 & VBA & BAV & VBA & BAV \\
5 & N & N & BAV & BVA \\
6 & AVB & BVA & VAB & VBA \\
7 & VAB & VAB & BVA & VAB \\
\hline
\end{tabular}

Figure 4: Sequences sorted by duration by quintiles

Sequences $A B V$ and $A V B$ clearly drive to shorter sessions, as they are at the top of table 4 . In fact, this shows that banner $A$ is not really easy to use, and many users do not understand how to interact with it. In fact, as it is a non-classic format, users may be a disconcerted, and do not realize the aim of the banner that recommends activities (since it appers as the first banner, before the more classic $B$ and $V$ banners). The first conclusion we can raise is that if the sequence starts with a "bad" banner, then the rest of the banner sequence is negatively affected.

On the contrary, sequences $V A B$ and $B V A$ lead to a longer session.

Another observation concerns the time when the first banner is injected (after the first decrease of activity). The average time is 30.6 seconds and we think it is too close from the beginning of the session: we may want the stimulation to lenghten the duration of the session, and adding the banner at the beginning will not have any effects.

We decided to conduct a second test round: the Analyzer was configured to insert the first banner upon the first activity decrease 150 seconds after the beginning of the session. This time was chosen using data collected during the first round (we tried to add the banner just before the end of the session, when a user gets bored of the site), but we realized that it was too long, since sessions during this test often last less than 150 seconds.

Based on the previous "failure", we finally conducted the last test round: the Analyzer was configured to insert the first banner upon the first activity decrease 80 seconds after the beginning of the session. We compare the average duration of stimulated users (287 seconds) with unstimulated users (209 seconds). It shows that the time when the banner is injected on the page is important: if we choose a time close to the end of the session to inject banners, we can lenghten the duration of the session.

Two main conclusions outstand. First, starting the sequence with an improper banner format wipes out the positive effects of subsequent banners. Second, adding the banner at an optimal time helps to get the user more interested and have longer visits on the site.

\section{CONCLUSION AND PERSPECTIVES}

In this paper, we discussed about the usefulness of considering sequence and timing for improving the effectiveness of ad banners. To support our argument, we presented a software platform that enables to conduct multivariate tests on a website. Based on three main components, our platform can get the navigation context of a user, providing key information to a site owner to understand his advertisement policies. We conduct tests on real users on two different levels, and try to answer two main questions: what are the best banner sequences? and when should a banner be displayed? Results show that an improper format of a banner in the beginning of the sequence wipes out the positive effects of subsequent banners, and that adding the banner at the end of the session can lead to increase the interest of the user and lengthen his session duration.

Many possible extensions and enhancements of this work can be addressed. For example, other key parameters of banner deployment need to be studied, such as the optimal spatial placement. The closed-loop characteristic of our web adaptation system can provide a natural ground for developing dynamic advertisement strategies. We believe it is possible to build rich statistical models (e.g. Markov Decision Processes-based) that can discover by themselves optimal advertising strategies, highly contextualized, and therefore perform even better than heuristics designed by web marketers with the average user in mind.

\section{REFERENCES}

[1] R. Kohavi, R. M. Henne, and D. Sommerfield. Practical guide to controlled experiments on the web: listen to your customers not to the hippo. In $K D D$ ' 0 \%: Proceedings of the 13th ACM international conference on Knowledge discovery and data mining, pages 959-967, New York, NY, USA, 2007. ACM.

[2] A. A. Labroo and S. Ramanathan. The influence of experience and sequence of conflicting emotions on ad attitudes. Journal of Consumer Research, 33(4):523-528, 2007.

[3] M. D. Loda and B. C. Coleman. Sequence matters: A more effective way to use advertising and publicity. Journal of Advertising Research, 45(04):362-372, 2005.

[4] M. Madambath. The idea of sequential advertising moving beyond the context. www.watblog.com/2009/01/30/the-idea-of-sequentialadvertising-moving-beyond-the-context/.

[5] T. Mei, X.-S. Hua, and S. Li. Contextual in-image advertising. In $M M$ '08: Proceedings of the 16th ACM international conference on Multimedia, pages 439-448, New York, NY, USA, 2008. ACM.

[6] T. Oshiba, Y. Koike, M. Tabuchi, and T. Kamba. Personalized advertisement-duration control for streaming delivery. In $M M$ '02: Proceedings of the 10th ACM international conference on Multimedia, pages 21-28, New York, NY, USA, 2002. ACM.

[7] G. Rosenkrans. The creativeness and effectiveness of online interactive rich media advertising. Journal of Interactive Advertising, 9(2), 2009.

[8] S. S. Sundar and J. Kim. Interactivity and persuasion: Influencing attitudes with information and involvement. Journal of Interactive Advertising, 5(2), 2005. 